\title{
The effects of bamboo leaf extract on growth performance, antioxidant traits, immune function and lipid metabolism of weaning piglets
}

\author{
J. Zhang, J. Wang, L. Zhang and T. Wang ${ }^{1}$ \\ Nanjing Agricultural University, College of Animal Science and Technology \\ No. 6,Tongwei Road, Xuanwu District, Nanjing 210095, P.R. China
}

KEY WORDS: bamboo leaf extract, antioxidation, immunity, lipid metabolism, growth performance, weaning piglets

Received: 7 May 2012

Revised: 26 June 2013

Accepted: 2 September 2013

${ }^{1}$ Corresponding author: e-mail: tianwang@njau.edu.cn

\begin{abstract}
The aim of this study was to evaluate the effects of dietary supplementation of bamboo leaf extract (BLE) on the growth performance, antioxidant traits, immune function, and lipid metabolism of weaning piglets. After weaning at 21 days, two hundred and forty healthy piglets (Large White $\times$ Landrance) were randomly assigned to 4 treatments with 6 pens and 10 piglets per pen. The control group (Ctr) received a maize-soyabean based diet, and the test groups received the control diet plus $0.5 \%, 1 \%$, or $2 \%(w / w)$ BLE, respectively. The experiment was carried out for 5 weeks. At the end of it, average daily feed intake in the 1\% BLE group was decreased $(p<0.05)$. Plasma concentrations of malondialdehyde were decreased with supplementation of $1 \%$ and $2 \%$ BLE. Immunoglobulin G concentrations and lysozyme activity in plasma were significantly increased in piglets supplemented with BLE. Diets with $1 \%$ and $2 \%$ BLE increased $(p<0.05)$ plasma concentrations of low density lipoprotein-cholesterol and reduced $(P<0.01)$ high density lipoprotein-cholesterol levels. Higher triglyceride concentrations were observed in the $0.5 \%(P<0.01)$ and $2 \%(p<0.05)$ BLE groups. In conclusion, these novel findings demonstrate that supplementation of BLE to the diet improved the antioxidant activity, immune function, and lipid metabolism of weaning piglets.
\end{abstract}

\section{Introduction}

Early weaning of piglets is often accompanied by severe growth retardation, anorexia, diarrhoea, poor performance, dysfunction of the immune system, and by higher morbidity (Lallès et al., 2007). Studies over the last decades have shown that dramatic changes in the composition of feed and in gastrointestinal function, together with other factors, including environmental ones, could be responsible for the growth inhibition (Kenworthy and Allen,
1966; Mormède and Hay, 2003). Thus, studies on the weaning process of piglets and development of novel strategies to minimize both morbidity and mortality of post-weaning piglets are crucial.

Bamboo is a group of evergreen plant genera belonging to the grass family, that are widely distributed in tropical and subtropical areas. In ancient China, bamboo was used for handicraft items, while its leaves served as a medicine for detoxification and fever. During the last few years, some of the bioactive constituents and potential health benefits 
of bamboo leaves have been identified. Bamboo leaf extracts contain mainly flavone glycosides, polysaccharides, amino acids, trace minerals, essential oils, chlorophyll and phenolic acids (Suga et al., 2003; Lu et al., 2005, 2006; Zhang et al., 2008). Polyphenolrich bamboo leaf extracts have been reported to exhibit various pharmacological properties, including free-radical scavenging, anti-oxidant, antibacterial, and antitumor activities, as well as prevention of lipid peroxidation and cancer (Kweon et al., 2001; Ren et al., 2004; Fujimura et al., 2005; Hasegawa et al., 2008; Lee et al., 2008a, 2010; Seki et al., 2010b).

Compared with synthetic nutraceuticals, which have caused undesirable side effects, bamboo leaf extract is predicted to be much safer by the virtue of its natural origin. According to the safety evaluation of bamboo leaves as an antioxidant, the maximum tolerated oral dose in rats is more than $10 \mathrm{~g} \cdot \mathrm{kg}^{-1}$, which places it in the non-poisonous category ( $\mathrm{Lu}$ et al., 2005). Moreover, bamboo leaf extract has been approved as a novel natural food additive by the Food Additive Standardization Committee of the People's Republic of China (PRC) in 2003 and has been listed in the state standard (GB2760-1996, Hygienic Standards for Food Additives in Use) since 2004 by the Ministry of Health of the PRC. Based on the safety and health-promoting effects of bamboo leaf extract, we hypothesize that it may serve as an effective feed additive for improving the health of pigs, specially weaning piglets. Therefore, the aim of this study was to investigate the effects of BLE on growth performance, antioxidant traits, immune function, and lipid metabolism of weaning piglets. Malondialdehyde (MDA) is a well-known carbonyl product of lipid peroxidation, and total superoxide dismutase (T-SOD) is a principal antioxidant enzyme, which catalyzes dismutation of the superoxide radical. High-density lipoprotein cholesterol (HDL-C), together with low-density lipoprotein cholesterol (LDL-C), comprise the largest fraction of serum cholesterol and play an important role in maintaining serum cholesterol homeostasis. Serum levels of such immunoglobulins as immunoglobulin $\mathrm{G}(\mathrm{IgG})$ and immunoglobulin A ( $\operatorname{Ig} \mathrm{A})$ provide key information on immune status. Thus, in the present study, all of the above-mentioned parameters were determined to evaluate the beneficial effects of BLE in weaning piglets.

\section{Material and method}

\section{Bamboo leaf extract (BLE)}

The BLE used in the present study was provided by Shanghai HanHuang Company Ltd. (Shanghai, PRC). It is made from fresh leaves and small branches of bamboo, Phyllostachys pubescens, through ethanol/ water extraction. The flavonoid concentration in BLE was $2.5 \%$, as determined by liquid chromatographmass spectrometer (LC-MS) analysis.

\section{Animal care and experimental design}

Two hundred and forty male piglets (Large White $\times$ Landrance) weaned at 21 days of age, $6.4 \pm 0.51 \mathrm{~kg}$ liveweight, were used in this study. The piglets were randomly allotted to 4 groups with 6 replicates and 10 piglets perpen. Piglets in 4 groups were fed the basal diet containing $0 \%(\mathrm{w} / \mathrm{w}) \mathrm{BLE}$ (control group), $0.5 \%(\mathrm{w} / \mathrm{w})$ BLE, $1.0 \%(\mathrm{w} / \mathrm{w})$ BLE, or $2.0 \%(\mathrm{w} / \mathrm{w})$ BLE, respectively. All piglets were allowed ad libitum access to feed and water and housed in a temperature- and humiditycontrolled environment with a $12 \mathrm{~h}$ light-dark cycle. The diets were formulated based on the NRC (1998) to meet the nutrient requirements of weaning piglets (Table 1).

Table 1. Composition of basal diets fed to weanling pigs

\begin{tabular}{lc}
\hline Indices & 1 to $35 \mathrm{~d}$ \\
\hline Ingredient, \% & 63 \\
maize & 4 \\
soyabean meal & 25 \\
wheat bran & 4 \\
$\quad$ fish meal & 4 \\
$\quad$ premix & \\
Analysed nutrient content, \% of DM & \\
$\quad$ digestible energy, $\mathrm{MJ} \mathrm{kg}^{-1}$ & 13.60 \\
crude protein & 19.30 \\
$\quad$ calcium & 0.93 \\
available phosphorus & 0.52 \\
Lys & 1.00 \\
Met + Cys & 0.46 \\
\hline
\end{tabular}

${ }^{1}$ Premix provided per kg of diet: mg: $\mathrm{Cu}$ (as copper sulphate) 250, Fe (as ferrous sulphate) 130, Zn (as zinc sulphate) 130, Mn 60, Se 0.3 , I 0.4; IU: vit. A 8000 , vit. E 30 ; mg: vit. $K_{3} 3.56$, vit. $B_{1} 1.8$, vit. $B_{2}$, vit. $B_{6} 1.26$, vit. $B_{12} 0.002$, folic acid 0.3 , biotin 0.44 , niacin 32 , choline 500 , antioxidant 50

The experiments described here were conducted in compliance with the Chinese regulations concerning the protection of experiment animals. The study protocol was approved in advance by the Ethics Committee of Nanjing Agricultural University, Nanjing (China). Care of the animals during the experiment was in accordance with the committee guidelines. At the end of the experiment, blood samples were randomly withdrawn from six piglets per pen by jugular venipuncture into heparin-treated 
tubes and the plasma was separated by centrifugation at $3000 \mathrm{~g}$ for $15 \mathrm{~min}$ at $4^{\circ} \mathrm{C}$. The plasma samples were then frozen at $-20^{\circ} \mathrm{C}$ for further assay.

\section{Growth performance}

Piglets were weighed individually and feed consumption per pen was measured at the end of the experiment. From these data, average daily gain (ADG), average daily feed intake (ADFI) and feed/gain ratio $(F / G)$ were calculated for the entire experiment.

Daily recordings were obtained for mortality, diarrhoea ratio, and faecal score $(0=$ solid bar or granule, $1=$ soft faeces with one shape, $2=$ sticky shapeless faeces and mixture of faeces and water, $3=$ shapeless, liquid faeces). The faecal scorings were performed by two persons jointly and during the experiments a total of three persons were involved.

diarrhoea index $=$ total scores of faeces/(total number of experimental piglets $\times$ experimental days)

diarrhoea ratio $=$ (number of piglets with diarrhoea)/(total number of experimental piglets $\times$ experimental days).

\section{Analysis of total malondialdehyde (MDA), total superoxide dismutase (T-SOD) and total antioxidative capacity (T-AOC)}

MDA in the plasma of the piglets was measured with an MDA analysis kit (Nanjing Jiancheng Bioengineering Institute, China) following the manufacturer's instructions. Briefly, the samples were treated with thiobarbituric acid (TBA), which produces a red compound with an absorption maximum at 532 $\mathrm{nm}$ in the presence of MDA (Zeng et al., 2008).

T-SOD activity in the plasma of the piglets was measured with a T-SOD analysis kit (Nanjing Jiancheng Bioengineering Institute, China). The method utilized a tetrazolium salt to quantify the superoxide radicals generated by xanthine oxidase and hypoxanthine (Zeng et al., 2008).

T-AOC of the plasma was measured with a T-AOC analysis kit (Nanjing Jiancheng Bioengineering Institute, China). The antioxidants in the samples can be utilized by the kit to reduce $\mathrm{Fe}^{3+}$ to $\mathrm{Fe}^{2+}$, which is then chelated with porphyrin to produce a purple complex; this complex can be quantified by measuring the absorbance at $550 \mathrm{~nm}$ (Liu et al., 2012b).

\section{Determination of immunoglobulin (IgA) and, (IgG), and lysozyme}

The IgG content in the plasma was measured with a radioimmunoassay kit from Beijing North Institute of Biological Technology (Beijing, China) (Liu et al., 2012a).
The plasma IgA content was measured with a Porcine IgA ELISA kit (BlueGene, Shanghai, China) following the manufacturer's instructions. In detail, the microtiter plates were coated with a monoclonal anti-IgA and a IgA-HRP conjugate. When incubated for $1 \mathrm{~h}$ at $37^{\circ} \mathrm{C}$, IgA from samples and IgA-HRP conjugate competed for the anti- IgA antibody binding site. After manual washing 3 to 5 times to remove the incubation mixture, the microtiter plates were inverted and dried. Substrates A and B were added to each well and incubated for $15 \mathrm{~min}$ at $20^{\circ}-25^{\circ} \mathrm{C}$. Finally, the stop solution was added to each well and the optical density was measured at $450 \mathrm{~nm}$. The IgA content in the plasma was determined by comparison with a standard curve.

Lysozyme activity was measured using a commercial kit (Nanjing Jiancheng Bioengineering Institute, China) that can utilize lysozyme in the samples to hydrolyse peptidoglycan of the bacterial cell wall. When the bacteria is lysed, the concentration of the bacterium in the liquid decreases, leading to a rise in the transmittance of the liquid. Transmittance was recorded at $530 \mathrm{~nm}$ at 5 and $125 \mathrm{~s}$ post-reaction. The lysozyme activity of the samples was determined by comparison with a standard.

\section{Analysis of triglyceride (TG), high density lipoprotein-cholesterol (HDL-C), low density lipoprotein-cholesterol (LDL-C) and total cholesterol (TC)}

The TG content of the plasma was measured with a TG analysis kit (Beijing BHKT Clinical Reagent Co. Ltd., China). The kit utilizes a reagent mix reagent that, in the presence of TG, produces quinones with an absorption maximum at $500 \mathrm{~nm}$. The reagentmix consists of lipoprotein lipases (LPL), glycerol kinase (GK), glycerol phosphate oxidase (GPO) and peroxidase (POD). The TG contents were determined by comparison with the calibrator (Trinder, 1969).

The HDL-C concentration in the plasma of piglets was measured with an LDL-C analysis kit (Beijing BHKT Clinical Reagent Co. Ltd., China) following the manufacturer's instructions. Briefly, samples were treated with phosphotungstic acid and the supernatant was collected. After reacting with a series of enzymes, absorbance of the supernatant was measured at $500 \mathrm{~nm}$. The HDL-C concentration was determined by comparison with a standard (Lopes-Virella et al., 1977).

The LDL-C concentration in the plasma of piglets was measured with an LDL-C analysis kit (Beijing BHKT Clinical Reagent Co. Ltd., China). Polylyethylene sulphate can selectively precipitate LDL from the plasma and leave very low density lipoprotein (VLDL) 
and high density lipoprotein (HDL) in the supernatant. After measuring the absorbance at $520 \mathrm{~nm}$, the cholesterol in the supernatant was determined by comparison with the calibrator. The LDL-C content was then quantified by subtracting the cholesterol in the supernatant from total cholesterol (Assmann et al., 1984).

The total cholesterol (TC) content of the plasma was measured with a TC analysis kit (Beijing BHKT Clinical Reagent Co. Ltd., China). The method utilized a mixed reagent to react with the samples, which in the presence of TG produced quinone compounds with an absorption maximum at $500 \mathrm{~nm}$. The reagent mixture consisted of CE, CO, POD, and 4-AAP. The TC content was determined by comparison with the calibrator (Trinder, 1969).

\section{Statistical analysis}

The data were analysed by one-way analysis of variance (ANOVA) using SPSS software (17.0). All data were expressed as mean \pm SEM. The difference between means was determined using Duncan's multiple range test at a level of significance of $p<0.05$.

\section{Results}

\section{Growth performance}

The effect of dietary supplementation of BLE on the growth performance of piglets is presented in Table 2. At the end of the experiment, the ADFI of piglets fed 1\% BLE was significantly reduced $(p<0.05)$, as compared with controls. Dietary BLE tended to decrease $\mathrm{F} / \mathrm{G}$, in particular, during the 1-14 d over the entire experimental period, $\mathrm{F} / \mathrm{G}$ was significantly reduced $(p<0.05)$ in the $2 \%$ BLE group.

\section{T-AOC and T-SOD activities, and MDA content}

Table 3 shows the effects of dietary BLE supplementation on plasma T-AOC, T-SOD activities, and MDA content in weaning piglets. Daily supplementation with $1.0 \%(p<0.05)$ and $2.0 \%(P<0.01)$ BLE resulted in a significant reduction in plasma MDA concentrations, but had no significant effect on T-AOC content and T-SOD activities in the plasma $(p>0.05)$.

\section{IgA and IgG concentrations, and lysozyme activity}

Table 4 shows IgA and IgG contents, and lysozyme activity in the plasma of piglets. Plasma lysozyme activity increased $(P<0.01)$ with increasing dietary BLE level. BLE supplementation in piglets significantly increased $(P<0.05)$ plasma IgG concentrations. No differences in plasma IgA concentrations were observed, however, in response to BLE supplementation.

\section{TG, HDL-C, LDL-C and TC concentrations}

The effects of dietary supplemental BLE on TG, HDL-C, LDL-C and TC concentrations are presented in Figure 1. Plasma HDL-C concentrations were significantly increased $(P<0.01)$ in both the $1 \%$ and $2 \%$ BLE groups, while plasma LDL-C concentrations were significantly reduced $(P<0.01)$ in all treatments. Plasma TG levels were significantly increased $(P<0.01)$ by feeding $0.5 \%$ and $2 \% \mathrm{BLE}$, as compared with controls. No significant differences were observed in plasma TC levels among the control and BLE groups.

Table 2. Effects of bamboo leaf extract on the growth performance of weaning piglets

\begin{tabular}{|c|c|c|c|c|c|}
\hline \multirow{2}{*}{$\begin{array}{l}\text { Period } \\
\text { days }\end{array}$} & \multirow{2}{*}{ Item } & \multicolumn{4}{|l|}{ Treatment } \\
\hline & & Ctr & $0.5 \%$ BLE & $1 \%$ BLE & $2 \%$ BLE \\
\hline & $A D G, g$ & $222.30 \pm 12.25$ & $228.60 \pm 14.43$ & $217.37 \pm 7.89$ & $252.00 \pm 9.87$ \\
\hline \multirow[t]{3}{*}{$1-14$} & $\mathrm{ADFI}, \mathrm{g}$ & $363.67 \pm 20.65$ & $369.70 \pm 17.05$ & $325.93 \pm 6.45$ & $348.73 \pm 20.56$ \\
\hline & $F / G$ & $1.64 \pm 0.06^{a}$ & $1.62 \pm 0.06^{a}$ & $1.50 \pm 0.03^{a b}$ & $1.38 \pm 0.03^{b}$ \\
\hline & $A D G, g$ & $334.77 \pm 21.60$ & $320.27 \pm 21.36$ & $274.13 \pm 22.92$ & $281.03 \pm 20.18$ \\
\hline \multirow[t]{3}{*}{$15-28$} & ADFI, g & $543.87 \pm 16.43$ & $539.67 \pm 51.50$ & $477.40 \pm 39.91$ & $495.43 \pm 58.61$ \\
\hline & $F / G$ & $1.63 \pm 0.07$ & $1.70 \pm 0.21$ & $1.74 \pm 0.07$ & $1.79 \pm 0.21$ \\
\hline & $A D G, g$ & $467.10 \pm 8.41$ & $499.83 \pm 55.19$ & $419.93 \pm 22.02$ & $428.73 \pm 43.72$ \\
\hline \multirow[t]{3}{*}{$29-35$} & $\mathrm{ADFI}, \mathrm{g}$ & $856.13 \pm 48.20$ & $804.63 \pm 57.12$ & $651.20 \pm 27.82$ & $741.13 \pm 19.40$ \\
\hline & $\mathrm{F} / \mathrm{G}$ & $1.83 \pm 0.10$ & $1.62 \pm 0.08$ & $1.56 \pm 0.13$ & $1.76 \pm 0.15$ \\
\hline & $A D G, g$ & $289.07 \pm 16.61$ & $301.83 \pm 2.23$ & $290.73 \pm 18.44$ & $281.77 \pm 10.90$ \\
\hline \multirow[t]{2}{*}{$1-35$} & ADFI, g & $534.23 \pm 23.30^{a}$ & $525.67 \pm 30.43^{a}$ & $451.40 \pm 18.37^{b}$ & $486.13 \pm 22.78^{\mathrm{ab}}$ \\
\hline & $F / G$ & $1.86 \pm 0.16$ & $1.74 \pm 0.11$ & $1.57 \pm 0.15$ & $1.72 \pm 0.03$ \\
\hline \multirow[t]{3}{*}{$1-35$} & diarrhoea rate, $\%$ & $3.47 \pm 0.17$ & $3.99 \pm 0.49$ & $5.38 \pm 0.76$ & $4.42 \pm 0.91$ \\
\hline & diarrhoea index & $0.11 \pm 0.00$ & $0.10 \pm 0.00$ & $0.11 \pm 0.01$ & $0.11 \pm 0.02$ \\
\hline & mortality, \% & $5.00 \pm 8.66$ & $8.33 \pm 10.41$ & $0.00 \pm 0.00$ & $5.26 \pm 9.12$ \\
\hline
\end{tabular}

a,b means within a row with no common superscripts differ significantly $(p<0.05)$; BLE - bamboo leaf extract; ADG - average daily gain; ADFI - average daily feed intake; F/G - feed/gain ratio; Ctr - control 
Table 3. Effects of bamboo leaf extract on the level of anti-oxidant in plasma of weaning piglets

\begin{tabular}{|c|c|c|c|c|}
\hline \multirow{2}{*}{ Indices } & \multicolumn{4}{|l|}{ Treatment $^{1}$} \\
\hline & Ctr & $0.5 \%$ BLE & $1 \% \mathrm{BLE}$ & $2 \% \mathrm{BLE}$ \\
\hline \multicolumn{5}{|l|}{$1-35 \mathrm{~d}$ after weaning } \\
\hline $\mathrm{T}-\mathrm{AOC}, \mathrm{U} \cdot \mathrm{ml}^{-1}$ & $6.02 \pm 1.35$ & $6.21 \pm 1.02$ & $6.47 \pm 0.86$ & $6.76 \pm 0.86$ \\
\hline $\mathrm{MDA}, \mathrm{nmol} \cdot \mathrm{ml}^{-1}$ & $3.37 \pm 0.23^{\mathrm{aA}}$ & $3.26 \pm 0.32^{\mathrm{abA}}$ & $3.01 \pm 0.22^{\mathrm{bA}}$ & $2.41 \pm 0.25^{\mathrm{cB}}$ \\
\hline $\mathrm{T}-\mathrm{SOD}, \mathrm{U} \cdot \mathrm{ml}^{-1}$ & $99.74 \pm 11.12$ & $95.58 \pm 5.73$ & $101.19 \pm 10.24$ & $99.64 \pm 9.47$ \\
\hline
\end{tabular}

a,b means within a row with no common superscripts differ significantly $(p<0.05) ;{ }^{1}$ see Table $2 ; \mathrm{A}, \mathrm{B}$ means within a row with no common super scripts differ significantly $(\mathrm{P}<0.01)$; T-AOC - total antioxidant capasity; MDA - total malondialdehyde; T-SOD - superoxide dismutase; Ctr - control

Table 4. Effects of bamboo leaf extract on immune function of weaning piglets

\begin{tabular}{|c|c|c|c|c|}
\hline \multirow{2}{*}{ Indices } & \multicolumn{4}{|l|}{ Treatment $^{1}$} \\
\hline & Ctr & $0.5 \%$ BLE & $1 \% \mathrm{BLE}$ & $2 \%$ BLE \\
\hline \multicolumn{5}{|l|}{$1-35 d$ after weaning } \\
\hline $\operatorname{lgG}, \mu \mathrm{g} \cdot \mathrm{ml}^{-1}$ & $0.34 \pm 0.13^{\mathrm{aA}}$ & $0.64 \pm 0.08^{\mathrm{bB}}$ & $0.63 \pm 0.05^{\mathrm{bB}}$ & $0.61 \pm 0.10^{\mathrm{bB}}$ \\
\hline $\lg \mathrm{A}, \mathrm{mg} \cdot \mathrm{ml}^{-1}$ & $19.61 \pm 8.87$ & $20.53 \pm 2.29$ & $22.06 \pm 2.53$ & $22.81 \pm 2.55$ \\
\hline lysozyme, $\mathrm{U} \cdot \mathrm{ml}^{-1}$ & $6.65 \pm 0.74^{\mathrm{aA}}$ & $8.32 \pm 0.54^{\mathrm{bB}}$ & $9.56 \pm 0.33^{c c}$ & $11.96 \pm 0.84^{\mathrm{dD}}$ \\
\hline
\end{tabular}

a,b, A,B as in Table 3; ${ }^{1}$ see Table 2; lgG - immunoglobulin G; IgA - immunoglobulin A; Ctr - control

A

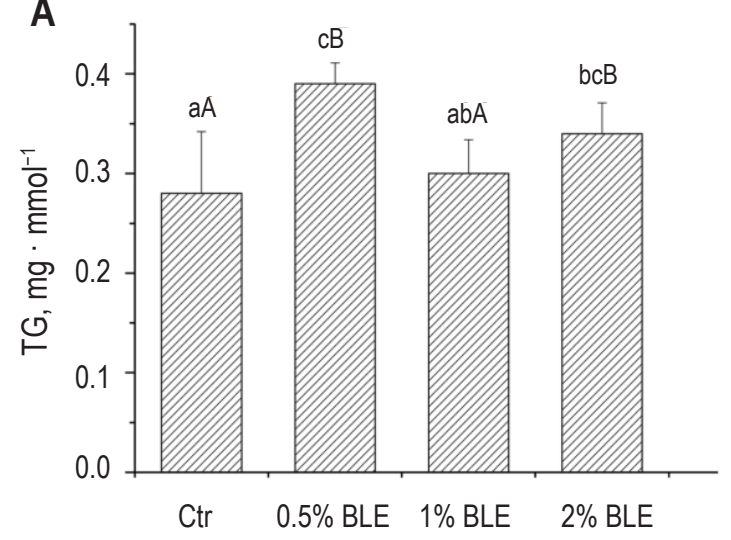

C

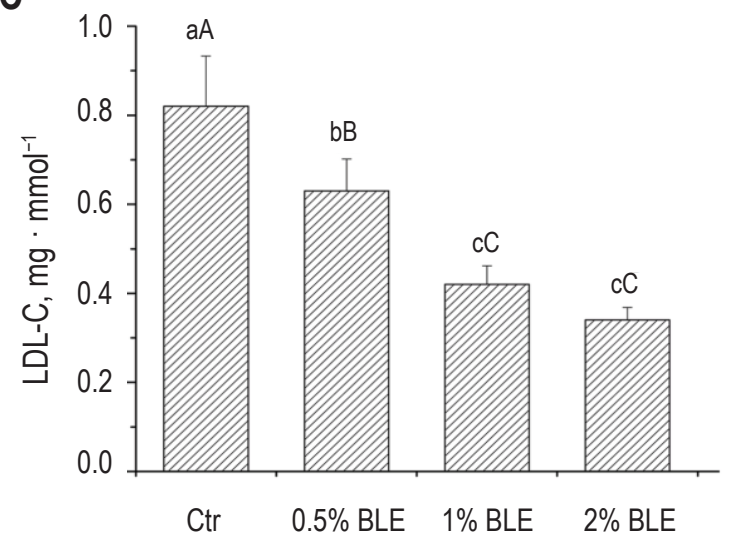

B

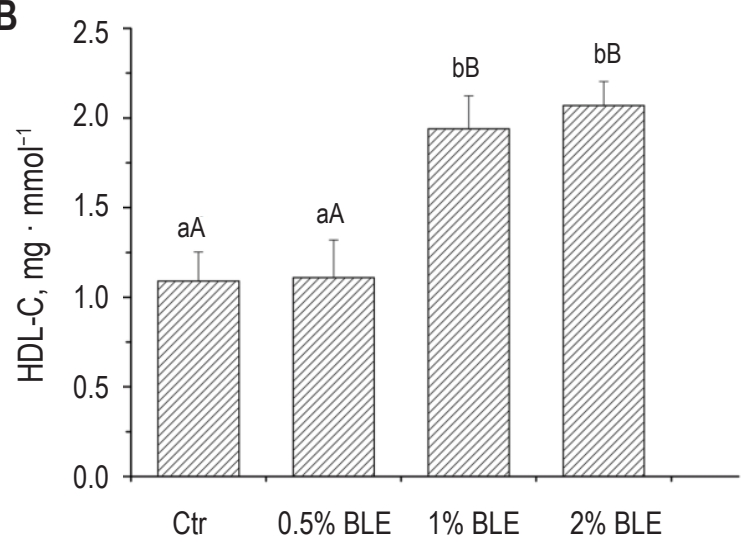

D

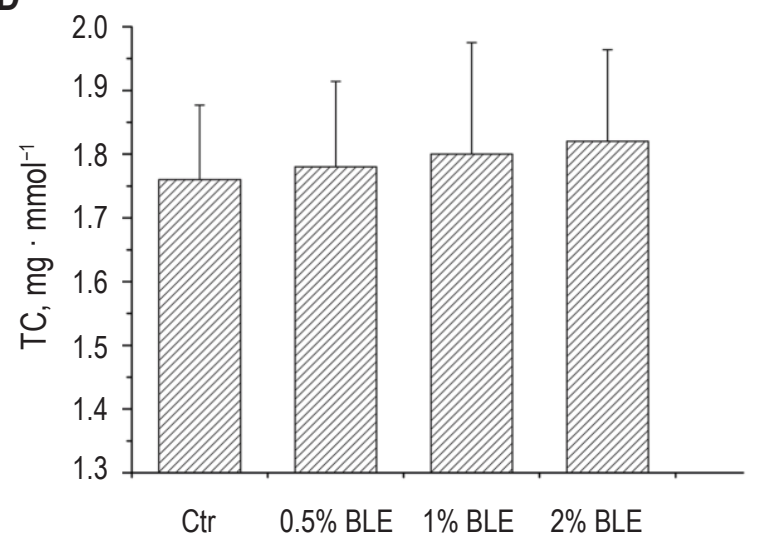

Figure 1. Effects of bamboo leaf extract on lipid metabolism in plasma of weaning piglets. Panels $A-D$ are bar graphs with BLE plotted against different concentrations of (A) triglyceride (TG), (B) high density lipoprotein-cholesterol (HDL-C), (C) low density lipoprotein-cholesterol $(\mathrm{LDL}-\mathrm{C})$, and $(\mathrm{D})$ total cholesterol (TC). Data are presented as mean \pm SEM $(n=6$ per group). $(a-d)$ or $(A-D)$ Data in the same row with a different superscripts letter differ significantly $(p<0.05$ or $P<0.01)$. Data were analysed by one-way analysis of variance (ANOVA); Ctr $-\operatorname{control}$ 


\section{Discussion}

In the past few decades, bamboo extracts have attracted greater interest as a dietary source of natural polyphenol anti-oxidants. Several lines of evidence have demonstrated the benefits of different parts of bamboo, such as bamboo leaves (Kobayashi et al., 1998; Lee et al., 2010), culm (Lee et al., 2008a,b), shoot (Fujimura et al., 2005; Park and Jhon, 2010), shavings (Zhang et al., 2004, 2006) and oils (Choi et al., 2008a; Cho et al., 2009). The present study shows that dietary bamboo leaf extract affected the growth performance, anti-oxidant traits, immune function, and lipid metabolism of weaning piglets. We found that ADFI of piglets fed BLE was significantly reduced and accompanied by no changes in ADG, which is consistent with other reports ( $\mathrm{Lu}$ et al., 2011). Due to the presence of essential oils and aromatic compounds in bamboo products, it is suggested they could render piglet diets unpalatable, leading to a pronounced reduction in ADFI. Our results also show that there were no changes in diarrhoea rate or index among various groups after administration of BLE. The presence of antimicrobial benzoic acid and 2,6-dimethoxybenzoquinine has, however, been reported in bamboo leaves and this restrained the proliferation of pathogenic bacterium in the gastrointestinal tract (Nishina et al., 1991). It is thought that numerous factors are associated with post-weaning diarrhoea of piglets, not only invasion of pathogenic microorganisms (Kenworthy and Allen, 1966; Moi et al., 1994) but also morphological and physiological changes in the small intestinal tract (Mormède and Hay, 2003). There are few studies that have reported on the effect of bamboo on the histomorphology of the gastrointestinal tract in vivo, which means there is no direct evidence showing whether bamboo-related products affect the gastrointestinal tract in animals. Moreover, due to the use of different animal models and variable purity of the extracts, it is difficult to perform accurate comparisons among the antibacterial activities of bamboo leaf extracts and other reported extracts in vivo or in vitro.

A great deal of attention has been paid to the anti-oxidant capacity of bamboo extracts. Here, we report that plasma MDA levels were significantly decreased by BLE supplementation, while there were only subtle changes in T-AOC activity. In agreement with our results, Lee et al. (2008a) also demonstrated that bamboo culm extract supplementation in mice fed an atherogenic diet significantly reduced the levels of thiobarbituric acid reactive substances and protein carbonyls, suggesting a protective effect of bamboo culm extract supplementation against lipid peroxidation and protein oxidation (Lee etal., 2008a). Results of a feeding test in vivo also showed that the anti-oxidants of bamboo leaves could significantly improve the activities of T-SOD and GPx (Zhang et al., 2007). But in the present study, it seems that BLE had no effect on the activity of TSOD. Compelling evidence supports the conclusion that the anti-oxidant capacity of plant materialsis due to their high polyphenol contents (Hanasaki et al., 1994; Velioglu et al., 1998; Bouaziz et al., 2004; Park and Jhon, 2010). Lu et al. (2005) reported that the anti-oxidants of bamboo leaves are a complex mixture, made up mostly of polyphenols. A recent report identified four flavone C-glycosides in bamboo leaf that were poorly absorbed in the gastrointestinal tract (Zhang et al., 2008). We believe that such poor absorption characteristics of flavone $\mathrm{C}$-glycosides in vivo indicated their potential benefits against oxidant damage in cells. In addition, a recent scientific paper focused on the anti-oxidant mechanism of bamboo extracts, which revealed that anti-oxidants from bamboo-protected cells against oxidative damage through induction of expression of phase II enzyme genes (Kweon et al., 2006). Furthermore, Koide et al. (2011) found that bamboo extracts showed lesser regulatory effects on Phase II enzymes in comparison with those on Phase I enzymes. In the modern environment, Phase I and II enzymes are vital for cell survival because they catalyse the conversion of xenobiotic or reactive free radicals into less toxic products via oxidation or conjugation and provoke the expression of Phase II detoxifying genes. Thus, enzyme-catalysed Phase I and II reactions may be an important detoxification pathway for bamboo leaf extract activity against oxidant stress.

It might be noteworthy that BLE plays an important role in the specific and non-specific immunity of organisms. Lysozyme is a natural enzyme produced by animals and is widely distributed in serum and tissues. Lysozyme has bactericidal activity and can be an opsonin that activates the complement system and phagocytes to prevent infection and disease (Jollès and Jollès, 1984; Geng et al., 2011). In the present study, the data showed that BLE significantly increased serum lysozyme activities, which indicates that BLE boosts the non-specific immune system. IgG and IgA are the largest and second-largest fractions among the serum immunoglobulins, respectively. Both play an important role in the function of an efficient immune system (Schroeder and Cavacini, 2010). In the current study, the IgG level was increased in the serum of 
piglets that received BLE supplementation, but no significant changes occurred in the IgA levels, suggesting an immune-stimulating activity of BLE. Results from Tsunoda et al. (1998) strongly suggested the potential of bamboo leaf extract as a tumor suppressive and cancer preventive food additive. It was reported that both in vitro and in vivo, bamboo leaf extracts increased the immunopotentiation effect through induction of the cytotoxic activity of NK cells and macrophages (Seki et al., 2010a). Thus, it is suggested that the effect on immune function of BLE is positively related to the increased activity of cytokines and secretion of immunoglobulins.

Numerous studies have demonstrated that bamboo leaf extract has beneficial effects on lipid profiles both in in vivo and in vitro models. In the present study, we found that HDL-C concentration were significantly increased with increasing concentration of BLE, whereas the effects of BLE on triglycerides were inconsistent. Studies on male Sprague-Dawley rats showed similar results: bamboo oil supplementation significantly reduced the TC and TG concentrations in blood and increased the plasma levels of HDL-C (Cho et al., 2009). Generally, high-density lipoprotein is one of the major carriers of cholesterol in the blood (McGrowder et al., 2010). Circulating HDL-C is regarded as 'good cholesterol', which plays a crucial role in maintaining cholesterol homeostasis between blood and peripheral tissues (Brown and Goldstein, 1986; Cho et al., 2009). In the present study, when the BLE concentration increased from $0.5 \%$ to $2.0 \%$, the HDL-C concentration increased from 1.11 to $2.07 \mathrm{mmol}$. $1^{-1}$, which was an increase of about $89.91 \%$ compared with the control group without addition of BLE. Nonetheless, we failed to find any significant difference in the TC levels among all groups, which is contrary to previous reports (Lee et al., 2008a; Lu et al., 2011).

On the other hand, our study has shown that the plasma LDL-C concentration linearly declined, accompanied by increasing BLE concentrations. The LDL-C concentration is controlled by the low density lipoprotein-acceptor number (Brown and Goldstein, 1986). Cho et al. (2009) indicated that bamboo oil effectively decreased the cholesterol concentration via inhibition of 3-hydroxy-3-methylglutaryl coenzyme A reductase activity, which is a major regulatory enzyme of hepatic cholesterol biosynthesis, and reduction of low-density lipoprotein-acceptor numbers. Quite recently, Higa and Panee (2011) found evidence of a strong inverse association between BLE concentration and nuclear factor kappa B
(NF-kB) activity. Hypercholesterolaemia or obesity are often accompanied by a state of chronic low-grade inflammation, characterized by the increasing release of pro-inflammatory cytokines such as interleukin 6 . It was reported that BLE attenuates the secretion of interleukin 6 through the suppression of the NF- $\mathrm{kB}$ signaling pathway (Higa and Panee, 2011). Modulation of interleukin 6 in high-fat diet-induced obese mice by BLE was reported to improve insulin resistance, which in turn improved the lipid profile (Yang et al., 2010). Accordingly, we speculate that the lipidlowering effect of bamboo leaf extract might partly be attributed to the modulation of inflammatory cytokines through the NF- $\mathrm{KB}$ signaling pathway.

\section{Conclusions}

Our results showed that bamboo leaf extract significantly reduced the malondialdehyde level and low-density lipoprotein cholesterol concentration in the plasma while increasing the high-density lipoprotein cholesterol (HDL-C) concentration and immunoglobulin $\mathrm{G}$ level. In particular, the dramatic increase in the concentration of HDL-C indicates a potential possibility for the bamboo leaf extracts used as a dietary supplement to improve lipid profiles in the animal industry. Further studies are still needed, however, to identify the bioactive constituents and elucidate the mechanism of bamboo leaf extract as a functional additive.

\section{Acknowledgements}

There were no conflicts of interest in the research. The author thank Jianjun Wang and Yuxiang Yang for the excellent technical assistance.

\section{References}

Assmann G., Jabs H., Kohnert U., Nolte W., Schriewer H., 1984. LDLcholesterol determination in blood serum following precipitation of LDL with polyvinylsulfate. Clin. Chim. Acta 140, 77-83

Bouaziz M., Chamkha M., Sayadi S., 2004. Comparative study on phenolic content and antioxidant activity during maturation of the olive cultivar Chemlali from Tunisia. J. Agr. Food Chem. $52,5476-5481$

Brown M.S., Goldstein J.L., 1986. A receptor-mediated pathway for cholesterol homeostasis. Science 232, 34-47

Cho H., Cho K.A., Jia S., Cho S.J., Choi D.B., 2009. Influence of bamboo oil supplementation on blood lipid concentration in serum. J. Ind. Eng. Chem.15, 281-284

Choi D.B., Cho K.A., Na M.S., Choi H.S., Kim Y.O., Lim D.H., Cho S.J., Cho H., 2008a. Effect of bamboo oil on antioxidative activity and nitrite scavenging activity. J. Ind. Eng. Chem. 14, 765-770 
Fujimura M., Ideguchi M., Minami Y., Watanabe K., Tadera K., 2005. Amino acid sequence and antimicrobial activity of chitinbinding peptides, Pp-AMP 1 and Pp-AMP 2, from Japanese bamboo shoots (Phyllostachys pubescens). Biosci. Biotechnol. Biochem. 69, 642-645

Hanasaki Y., Ogawa S., Fukui S., 1994. The correlation between active oxygens scavenging and antioxidative effects of flavonoids. Free Radical Biol. Med. 16, 845-850

Hasegawa T., Tanaka A., Hosoda A., Takano F., Ohta T., 2008. Antioxidant C-glycosyl flavones from the leaves of Sasa kurilensis var gigantea. Phytochemistry 69, 1419-1424

Higa J.K., Panee J., 2011. Bamboo extract reduces interleukin 6 (IL-6) overproduction under lipotoxic conditions through inhibiting the activation of NF-kB and AP-1 pathways. Cytokines 55, 18-23

Kenworthy R., Allen W., 1966. Influence of diet and bacteria on small intestinal morphology, with special reference to early weaning and Escherichia coli: studies with germfree and gnotobiotic pigs. J. Comp. Pathol. 76, 291

Kobayashi T., Tsunoda S., Inoue H., lijima K., Toriizuka K., Cyong J.C., Nagasawa H., 1998. Effects of Sasa Health, extract of bamboo grass leaves, on the thymic involution and thymocyte phenotypic alterations in SHN female mice. Bull. Fac. Agr. Meiji Univ. 115, 39-44

Koide C.L.K., Collier A.C., Berry M.J., Panee J., 2011. The effect of bamboo extract on hepatic biotransforming enzymes-Findings from an obese-diabetic mouse model. J. Ethnopharmacol. 133, 37-45

Kweon M.H., Hwang H.J., Sung H.C., 2001. Identification and antioxidant activity of novel chlorogenic acid derivatives from bamboo (Phyllostachys edulis). J. Agr. Food Chem. 49, 4646-4655

Kweon M.H., In Park Y., Sung H.C., Mukhtar H., 2006. The novel antioxidant 3-O-caffeoyl-1-methylquinic acid induces Nrf2dependent phase II detoxifying genes and alters intracellular glutathione redox. Free Radical Biol. Med. 40, 1349-1361

Lallès J.P., Bosi P., Smidt H., Stokes C.R., 2007. Weaning-A challenge to gut physiologists. Livest. Sci. 108, 82-93

Lee H.J., Kim K.A., Kang K.D., Lee E.H., Kim C.Y., Um B.H., Jung S.H., 2010. The compound isolated from the leaves of Phyllostachys nigra protects oxidative stress-induced retinal ganglion cells death. Food Chem. Toxicol. 48, 1721-1727

Lee M.J., Kim M.J., Song Y.S., Song Y.O., Moon G.S., 2008a. Bamboo culm extract supplementation elevates HDL-cholesterol and ameliorates oxidative stress in C57BL/6 mice fed atherogenic diet. J. Med. Food 11, 69-77

Lee M.J., Park W.H., Song Y.S., Lee Y.W., Song Y.O., Moon G.S., 2008b. Effect of bamboo culm extract on oxidative stress and genetic expression: Bamboo culm extract ameliorates cell adhesion molecule expression and NF-KB activity through the suppression of the oxidative stress. Clin. Nutr. 27, 755-763

Liu J., Chen D., Yao Y., Yu B., Mao X., He J., Huang Z., Zheng P., 2012a. Intrauterine growth retardation increases the susceptibility of pigs to high-fat diet-induced mitochondrial dysfunction in skeletal muscle. PLOS ONE 7, e34835

Liu J., Yao Y., Yu B., Mao X., Huang Z., Chen D., 2012b. Effect of folic acid supplementation on hepatic antioxidant function and mitochondrial-related gene expression in weanling intrauterine growth retarded piglets. Livest. Sci. 146, 123-132

Lopes-Virella M.F., Stone P., Ellis S., Colwell J., 1977. Cholesterol determination in high-density lipoproteins separated by three different methods. Clin. Chem. 23, 882-884
Lu B., Cai H., Huang W., Wu X., Luo Y., Liu L., Zhang Y., 2011. Protective effect of bamboo shoot oil on experimental nonbacterial prostatitis in rats. Food Chem. 124, 1017-1023

Lu B., Wu X., Shi J., Dong Y., Zhang Y., 2006. Toxicology and safety of antioxidant of bamboo leaves. Part 2: Developmental toxicity test in rats with antioxidant of bamboo leaves. Food Chem. Toxicol. 44, 1739-1743

Lu B., Wu X., Tie X., Zhang Y., 2005. Toxicology and safety of antioxidant of bamboo leaves. Part 1: Acute and subchronic toxicity studies on anti-oxidant of bamboo leaves. Food Chem. Toxicol. 43, 783-792

McGrowder D., Riley C., Morrison E.Y.St.A., Gordon L., 2011. The role of high-density lipoproteins in reducing the risk of vascular diseases, neurogenerative disorders, and cancer. Cholesterol 2011, 1-9

Moi P., Chan K., Asunis I., Cao A., Kan Y.W., 1994. Isolation of NF-E2related factor 2 (Nrf2), a NF-E2-like basic leucine zipper transcriptional activator that binds to the tandem NF-E2/AP1 repeat of the beta-globin locus control region. Proc. Natl. Acad. Sci. USA 91, 9926-9930

Mormède P., Hay M., 2003. Behavioural changes and adaptations associated with weaning. In: J.L. Dividich, J.R. Pluske, MWA. Verstegen (Editors). Weaning the Pig: Concepts and Consequences. Wageningen Academic Publisher (the Netherlands), pp. 53-60

Nishina A., Hasegawa K., Uchibori T., Seino H., Osawa T., 1991. 2, 6-Dimethoxy-p-benzoquinone as an antibacterial substance in the bark of Phyllostachys heterocycla var. pubescens, a species of thick-stemmed bamboo. J. Agr. Food Chem. 39, 266-269

NRC, 1998. Nutrient Requirements of Swine. $10^{\text {th }}$ revised Edition. National Academy of Science Press. Washington, DC

Park E.J., Jhon D.Y., 2010. The antioxidant, angiotensin converting enzyme inhibition activity, and phenolic compounds of bamboo shoot extracts. LWT-Food.Sci. Tech. 43, 655-659

Ren M., Reilly R.T., Sacchi N., 2004. Sasa health exerts a protective effect on Her2/NeuN mammary tumorigenesis. Anticancer Res. 24, 2879-2884

Seki T., Kida K., Maeda H., 2010a. Immunostimulation-mediated antitumor activity of bamboo (Sasa senanensis) leaf extracts obtained under vigorous condition. Evid-Based Compl. Alt. 7, 447-457

Seki T., Maeda H., 2010b. Cancer preventive effect of kumaizasa bamboo leaf extracts administered prior to carcinogenesis or cancer inoculation. Anticancer Res. 30, 111-118

Suga A., Takaishi Y., Goto S., Munakata T., Yamauchi I., Kogure K., 2003. Two lignan dimers from bamboo stems (Phyllostachys edulis). Phytochemistry 64, 991-996

Trinder P., 1969. Determination of glucose concentration in the blood. Ann. Clin. Biochem. 6, 24

Tsunoda S., Yamamoto K., Sakamoto S., Inoue H., Nagasawa H., 1998. Effects of Sasa Health, extract of bamboo grass leaves, on spontaneous mammary tumourigenesis in SHN mice. Anticancer Res. 18, 153-158

Velioglu Y., Mazza G., Gao L., Oomah B., 1998. Antioxidant activity and total phenolics in selected fruits, vegetables, and grain products. J. Agr. Food Chem. 46, 4113-4117

Yang J.H., Lim H.S., Heo Y.R., 2010. Sasa borealis leaves extract improves insulin resistance by modulating inflammatory cytokine secretion in high fat diet-induced obese C57/BL6J mice. Nutr. Res. Pract. 4, 99-105 
Zeng T., Zhang C.L., Zhu Z.P., Yu L.H., Zhao X.L., Xie K.Q., 2008. Diallyl trisulfide (DATS) effectively attenuated oxidative stressmediated liver injury and hepatic mitochondrial dysfunction in acute ethanol-exposed mice. Toxicology 252, 86-91

Zhang Y., Jiao J., Liu C., Wu X., 2008. Isolation and purification of four flavone C-glycosides from antioxidant of bamboo leaves by macroporous resin column chromatography and preparative high-performance liquid chromatography. Food Chem. 107, 1326-1336
Zhang Y., Tie X., Bao B., Wu X., 2007. Metabolism of flavone C-glucosides and $p$-coumaric acid from antioxidant of bamboo leaves (AOB) in rats. Brit. J. Nutr. 97, 484-494

Zhang Y., Wu X., Ren Y., Fu J., 2004. Safety evaluation of a triterpenoid-rich extract from bamboo shavings. Food Chem. Toxicol. 42, 1867-1875

Zhang Y., Yao X., Bao B., 2006. Anti-fatigue activity of a triterpenoidrich extract from Chinese bamboo shavings (Caulis bamfusae in taeniam). Phytother. Res. 20, 872-876 